\title{
Mortality Following Distal Femur Fractures Versus Proximal Femur Fractures in Elderly Population: The Impact of Best Practice Tariff
}

\author{
Islam Mubark $^{1}$, Amr Abouelela ${ }^{1}$, Ahmed Genena ${ }^{2,} 3$, Abdallah Al Ghunimat ${ }^{1}$, Islam Sarhan ${ }^{1,4}$, Neil
} Ashwood $^{1}$

1. Trauma and Orthopaedics, University Hospitals of Derby and Burton NHS Foundation Trust, Derby, GBR 2. Trauma and Orthopaedics, James Paget University Hospitals NHS Foundation Trust, Norwich, GBR 3. Trauma and Orthopaedics, Faculty of Medicine, Helwan University, Helwan, EGY 4. Trauma and Orthopaedics, Faculty of Medicine, Alexandria University, Alexandria, EGY

Corresponding author: Islam Mubark, islam.mubark@nhs.net

\section{Abstract}

\section{Background and objectives}

The mortality after hip, proximal femur, fractures in elderly patients has steadily declined in the last decade in the United Kingdom as a result of implementing of multiple protocols focusing on prompt multidisciplinary pre- and post-operative optimization and reducing time to surgery. The pinnacle of these protocols is the development of the best practice tariff as an incentive program for hospitals that meet set criteria by the National Health Service (NHS) England in managing these injuries. Until the time of writing this paper, there was no parallel program for the management of fractures involving distal femur in the elderly. The aim of this study is to evaluate the outcomes of distal femur fractures in elderly patients against proximal femur fractures regarding post-injury mortality, the prevalence of surgical treatment and time delay till surgery.

\section{Methods}

A retrospective study of all patients above the age of 60 admitted to Queens Hospital Burton between 2010 and 2014 with fractures involving distal end of the femur. Patient data were assessed for demographic criteria, co-morbidities as per Charleston Comorbidities Index, type of management, time-lapse before surgery and 30-day, six-month and one-year mortality. Results were compared to an age-matched control group of patients with proximal femur fractures randomly selected during the same time window.

\section{Results}

The main demographic criteria such as age, gender, and Charleston Comorbidities Index were similar in both groups. There were more patients treated non-operatively in the distal femur group than in the proximal femur group (15\% vs $4 \%$ ). Time to surgery was statistically significantly longer in distal femur group compared to the proximal femur (49.130 hours vs 34.075 hours, $\mathrm{P}=0.041)$. The mortality in distal femur group was higher at all times (9.68\% at 30 days, $20.32 \%$ at six months and $34.41 \%$ at one year) when

Review began 09/21/2020 Review ended 09/29/2020 Published 09/30/2020

\section{() Copyright 2020}

Mubark et al. This is an open access article distributed under the terms of the Creative Commons Attribution License CC-BY 4.0., which permits unrestricted use, distribution, and reproduction in any medium, provided the original author and source are credited. compared to that in the proximal femur group (6.99\% at 30 days, $14.52 \%$ at six months, $21.51 \%$ at one year).

\section{Conclusion}

The distal femoral fractures showed higher mortality at 30 days, six months and one year compared to the proximal femur group. This could be partly influenced by the implementation of best practice tariff in the proximal femur fracture group reflected in less time to surgery, pre- and post-operative multidisciplinary approach and more frequent operative management.

Categories: Emergency Medicine, Orthopedics, Trauma

Keywords: distal femur fracture, mortality rate, proximal femur fracture, best practice tariff, hip, elderly population, neck of femur fractures

\section{Introduction}

Hip fractures in the elderly have always been associated with a high rate of comorbidities and mortality with reported one-year mortality between $20 \%$ and $30 \%$ [1,2]. There is strong evidence that multidisciplinary approach in addition to reducing time to surgery is essential for reducing mortality in this frail patient group $[3,4]$. This has led the National Institute for Health and Care Excellence (NICE) in England to publish its guidance on hip fracture management emphasizing early surgery, pre- and post-operative optimization and multidisciplinary care [5]. In 2010, the National Health Service (NHS) improvement has implemented what is known as the best practice tariff. It is an incentive program that grants extra fund to hospitals that achieve certain targets in managing elderly patients with hip fracture. Amongst these targets are operating 
below 36 hours from the presentation and multidisciplinary approach including orthogeriatric review [6]. This approach has led to a significant reduction in 30-day-mortality in the United Kingdom in the last decade from $8.3 \%$ in 2009 to $6.1 \%$ in the year of 2018 according to the National Hip Fracture Database report 2019 [7].

Elderly patients sustaining distal femur fractures are usually frail and have multiple comorbidities. Mortality in this group of patients was reported being as high as in that following proximal femur fractures [8]. Until the time of conducting this study, the best practice tariff scheme only included proximal femur fractures which include intracapsular, intertrochanteric and subtrochanteric fractures, i.e. fractures within $5 \mathrm{~cm}$ distal to the lesser trochanter. There was no parallel best practice tariff for fracture femur sustained outside these anatomical areas. In this study, we assumed that elderly patients with distal femur fractures are similar in comorbidities to patients with proximal femur fractures. We also assumed that the lack of the best practice tariff may have led to delayed theatres and more frequent non-operative management, therefore higher mortality in the distal femur fracture group.

\section{Materials And Methods}

A retrospective study including all patients aged 60 years or more admitted to Queens Hospital Burton between 2010 and 2014. Inclusion criteria were closed fracture involving the lower third or distal end femur with or without extension to articular surface sustained following low energy trauma, i.e., simple fall from standing height. Exclusion criteria were pathologic, open and periprosthetic fractures.

Patients' records were reviewed for demographic criteria and comorbidities were scored using the Charleston Comorbidity Index (CCI).

An age-matched equal group of patients, admitted with proximal hip fractures (intracapsular, intertrochanteric and subtrochanteric), were randomly selected during the same time window as a control group.

Data were analyzed for the percentage of surgical treatment and time to surgery in each group. The primary outcome was mortality at 30 days, six months and one year.

\section{Results}

Each group included 189 patients. The mean age for distal femur group was 81.1 years (range 60-99 \pm standard deviation (SD) 8.1) and proximal femur was 81.8 years (range 61-101 \pm SD 8.9). Females were the predominant gender in both groups with $65 \%$ and $69 \%$ prevalence in distal and proximal femur groups, respectively.

Statistical analysis was performed using Statistical Package for the Social Sciences (SPSS) version 23 (IBM Corp., Armonk, NY). There was no statistically significant difference in the mean CCI with distal femur mean $\mathrm{CCI}$ of $(5.1 \pm \mathrm{SD} 2.1)$ against the mean of $(4.8 \pm \mathrm{SD} 2.2)$ in proximal femur group $(\mathrm{P}=0.612)$. The time-lapse till surgery in distal femur group (34.07 \pm SD 2.9 hours) was statistically significantly longer than proximal femur group (49.13 \pm SD 3.4 hours) $(\mathrm{P}=0.041)$ (Figure 1 ) (Table 1$)$.

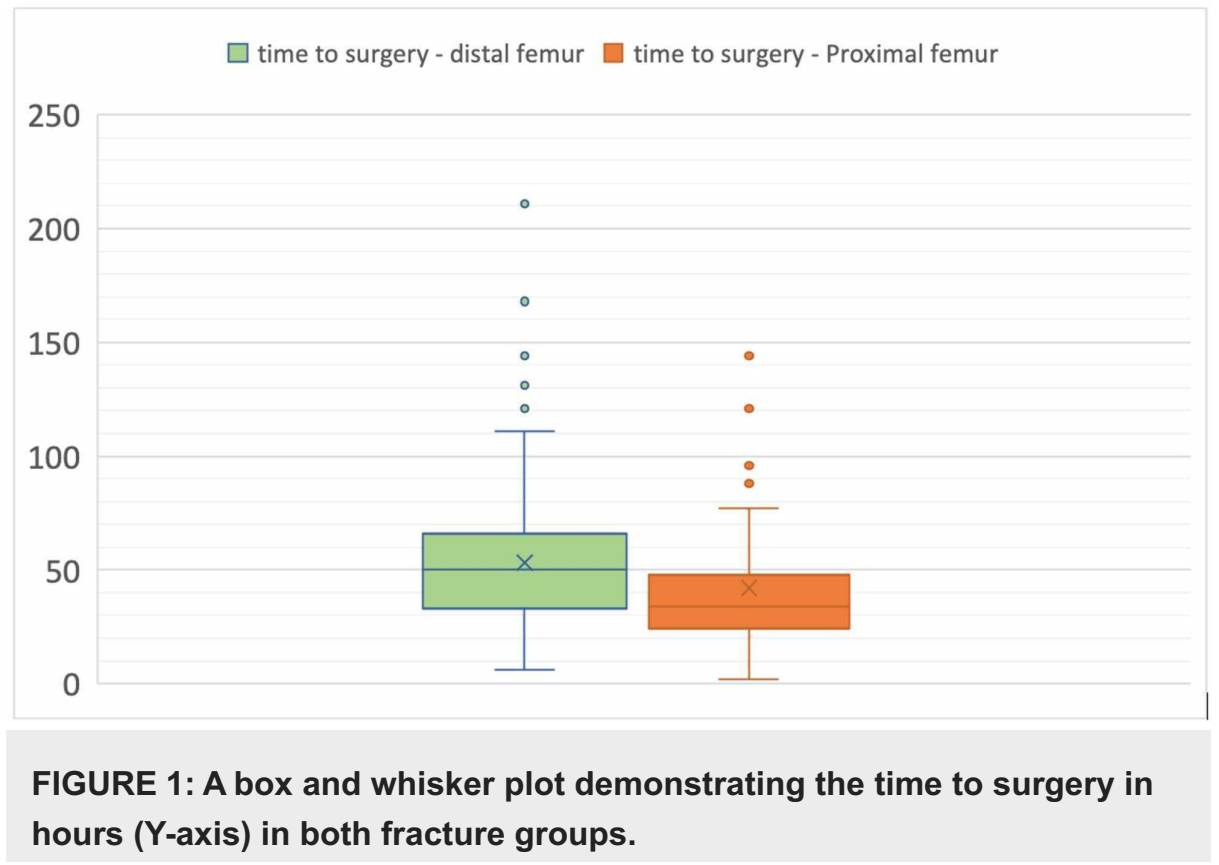




\section{Cureus}

\begin{tabular}{|c|c|c|c|}
\hline & $\begin{array}{l}\text { Distal femur fracture } \\
\text { group }\end{array}$ & $\begin{array}{l}\text { Proximal femur fracture group } \\
\text { (control) }\end{array}$ & \\
\hline Age in years Mean (range \pm standard deviation) & $81.1(60-99 \pm 8.1)$ & $81.8(61-101 \pm 8.9)$ & \\
\hline Females percentage & $65 \%$ & $69 \%$ & \\
\hline $\begin{array}{l}\text { Charleston Comorbidity Index (CCI) (mean } \pm \text { standard } \\
\text { deviation) }\end{array}$ & $5.1 \pm 2.1$ & $4.8 \pm 2.2$ & $\begin{array}{l}P= \\
0.612\end{array}$ \\
\hline Time to surgery in hours (mean \pm standard deviation) & $49.13 \pm 3.4$ & $34.07 \pm 2.9$ & $\begin{array}{l}P= \\
0.041\end{array}$ \\
\hline Percentage of surgery done within less than 36 hours & $46 \%$ & $69 \%$ & \\
\hline Percentage of non-operative management & $15 \%$ & $4 \%$ & \\
\hline
\end{tabular}

TABLE 1: Difference in the two groups in terms of demographics, Charleston Comorbidity Index, time to surgery, percentage of surgery done within less than $\mathbf{3 6}$ hours and percentage of nonoperative management.

Only $46 \%$ of the distal femur group had surgery under 36 hours from admission versus $69 \%$ in the proximal femur group. There were more patients treated non-operatively in distal femur group (15\%) compared to (4\%) in the proximal femur group. The non-operative management was selected in distal femur group either as a result of undisplaced fracture which could be managed without operation in $25 \%$ or the patient was high risk for anaesthesia in the remaining $75 \%$.

The distal femur group had higher mortality at all times (9.68\% at 30 days, $20.32 \%$ at six months and $34.41 \%$ at one year), compared to the proximal femur (6.99\% at 30 days, $14.52 \%$ at six months, $21.51 \%$ at one year) (Figure 2).

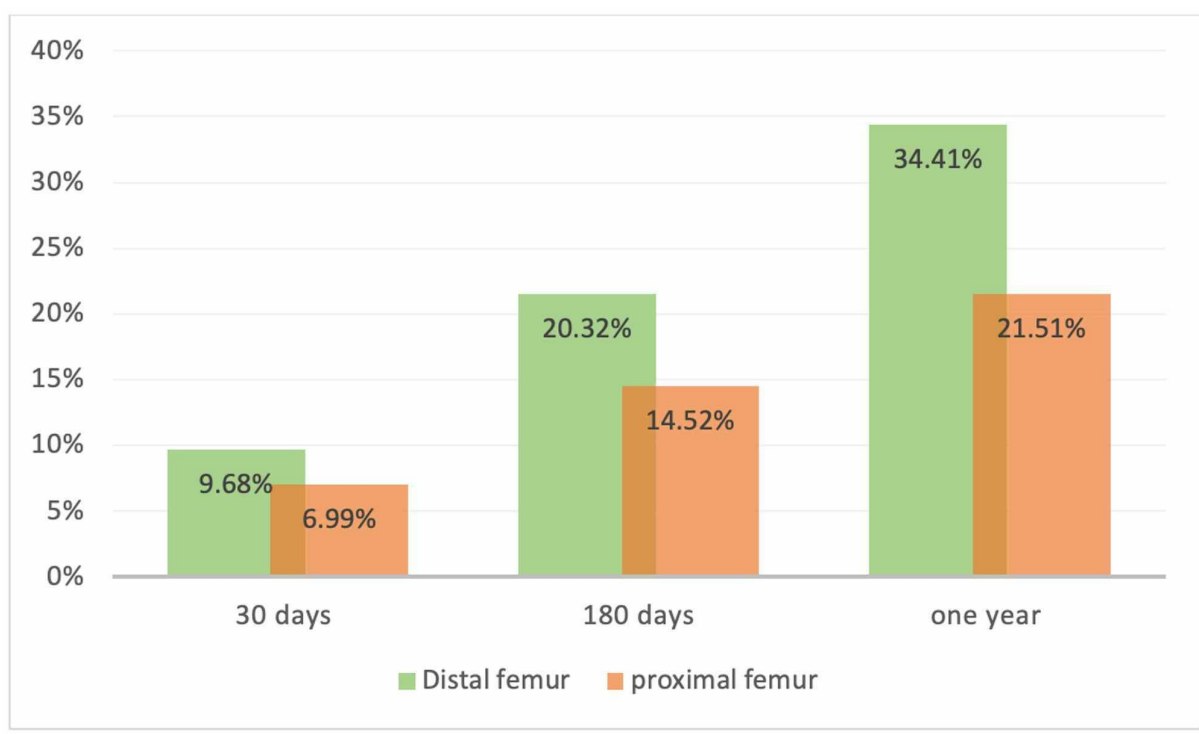

FIGURE 2: A bar chart demonstrating the mortality rate as a percentage (Y-axis) in both groups at 30 days, 180 days and one year ( $\mathrm{X}$-axis).

\section{Discussion}

Hip fractures in the elderly have been associated with high morbidity and mortality owing to the prevalence of medical comorbidities in this cohort of patients with reports of 30-day mortality as high as $9.6 \%$, while the one-year mortality rate increases to $33 \%[8,9]$. There has been a large body of evidence that multidisciplinary care and operating within the first 48 hours after injury reduce the incidence of both 
morbidity and mortality $[3,10]$. In 2010, the National Health Service (NHS) in the UK has implemented what is known as the best practice tariff which provides additional funding to hospitals if they meet certain goals in managing these patients. These targets are (1) surgery within 36 hours of presentation, (2) assessment by a geriatrician within 72 hours of admission, (3) an abbreviated mental test performed before surgery, (4) a nutritional assessment during the admission, (5) a delirium assessment during admission and (6) a physiotherapist assessment on the day of or day following surgery [11]. This tariff was applied to patients aged 60 or older with fractures limited to intracapsular, intertrochanteric and subtrochanteric, i.e., within 5 $\mathrm{cm}$ below the lesser trochanter. This approach has led to a reduction in the UK reported 30-day-mortality in the last decade from $8.3 \%$ in 2009 to $6.1 \%$ in the most recent 2019 national hip fracture database report with almost $70 \%$ receiving surgery within 36 hours from admission in the emergency department and only $2.2 \%$ of patient treated non-operatively [7].

Distal femur fractures are the second most frequent fractures of the femur in the elderly population following those of the hip and their management is challenged by the presence of multiple medical comorbidities [12]. One study reported $11 \%$ of women with a distal femur fracture to have osteoporosis, $21 \%$ of patients have diabetes and more than $32 \%$ have a cardiovascular disease [13]. Mortality rates in the elderly with distal femur fractures have been reported to be around $18 \%$ at six months and $18-30 \%$ at one year $[8,14,15]$.

In this study, we evaluated the outcome following fractures of the distal femur. The primary outcome was 30-day, six-month and one-year mortality against an age-matched control group of an equal number of proximal femur fractures' patients. The demographic criteria and comorbidities of both groups were comparable. We have been able to identify increased mortality at all times in the distal femur fracture group. This could be a combination of a higher percentage of time to surgery in this group in addition to more patients treated non-surgically. It is the authors' belief that the lack of best practice tariff cover for this group of patients could be a reason for these results and extending the best practice tariff to cover distal femur fractures could reduce the mortality in distal femur fracture close to proximal femur fracture. From April 2020, NHS England and NHS Improvement started the inclusion of distal femur fractures in the best practice tariff. It will be interesting to see how this would affect the mortality in this group of patients in the years to come.

\section{Conclusions}

Elderly population sustaining distal femur fractures shared the same co-morbidities to those sustaining proximal femur fractures. Nevertheless, the distal femoral fractures showed higher mortality at all times. This could be partly attributed to the implementation of the best practice tariff reflected in less time to surgery, multidisciplinary pre- and post-operative approach and a higher percentage of operative treatment in the proximal femur fracture group.

\section{Additional Information \\ Disclosures}

Human subjects: Consent was obtained by all participants in this study. Animal subjects: All authors have confirmed that this study did not involve animal subjects or tissue. Conflicts of interest: In compliance with the ICMJE uniform disclosure form, all authors declare the following: Payment/services info: All authors have declared that no financial support was received from any organization for the submitted work. Financial relationships: All authors have declared that they have no financial relationships at present or within the previous three years with any organizations that might have an interest in the submitted work. Other relationships: All authors have declared that there are no other relationships or activities that could appear to have influenced the submitted work.

\section{References}

1. Johnell O, Kanis JA: An estimate of the worldwide prevalence, mortality and disability associated with hip fracture. Osteoporos Int. 2004, 15:897-902. 10.1007/s00198-004-1627-0

2. Panula J, Pihlajamäki H, Mattila VM, Jaatinen P, Vahlberg T, Aarnio P, Kivelä S-L: Mortality and cause of death in hip fracture patients aged 65 or older - a population-based study. BMC Musculoskelet Disord. 2011, 12:105. 10.1186/1471-2474-12-105

3. Simunovic N, Devereaux PJ, Sprague S, Guyatt GH, Schemitsch E, DeBeer J, Bhandari M: Effect of early surgery after hip fracture on mortality and complications: systematic review and meta-analysis. CMAJ. 2010, 182:1609-1616. 10.1503/cmaj.092220

4. Hawley S, Kassim Javaid M, Prieto-Alhambra D, et al.: Clinical effectiveness of orthogeriatric and fracture liaison service models of care for hip fracture patients: population-based longitudinal study. Age Ageing. 2016, 45:236-242. 10.1093/ageing/afv204

5. NICE. Hip fracture: the management of hip fracture in adults . (2011). Accessed: September 9, 2020: https://www.nice.org.uk/guidance/cg124/evidence/full-guideline-183081997.

6. Chamberlain M, Pugh H: Improving inpatient care with the introduction of a hip fracture pathway . BMJ Qual Improv Rep. 2015, 4:204075-2786. 10.1136/bmjquality.u204075.w2786

7. National Hip Fracture Database (NHFD) annual report 2019. (2019). Accessed: September 4, 2020: https://www.rcplondon.ac.uk/projects/outputs/national-hip-fracture-database-nhfd-annual-report-2019. 


\section{Cureus}

8. Van Staa TP, Dennison EM, Leufkens HGM, Cooper C: Epidemiology of fractures in England and Wales . Bone. 2001, 29:517-522. 10.1016/S8756-3282(01)00614-7

9. Cenzer IS, Tang V, Boscardin WJ, et al.: One-year mortality after hip fracture: development and validation of a prognostic index. J Am Geriatr Soc. 2016, 64:1863-1868. 10.1111/jgs.14237

10. Grimes JP, Gregory PM, Noveck H, Butler MS, Carson JL: The effects of time-to-surgery on mortality and morbidity in patients following hip fracture. Am J Med. 2002, 112:702-709. 10.1016/S0002-9343(02)01119-1

11. Khan SK, Weusten A, Bonczek S, Tate A, Port A: The Best Practice Tariff helps improve management of neck of femur fractures: a completed audit loop. Br J Hosp Med. 2013, 74: 10.12968/hmed.2013.74.11.644

12. Karam J, Campbell P, David M, Hunter M: Comparison of outcomes and analysis of risk factors for nonunion in locked plating of closed periprosthetic and non-periprosthetic distal femoral fractures in a retrospective cohort study. J Orthop Surg Res. 2019, 14:150. 10.1186/s13018-019-1204-Z

13. Nieves JW, Bilezikian JP, Lane JM, Einhorn TA, Wang Y, Steinbuch M, Cosman F: Fragility fractures of the hip and femur: incidence and patient characteristics. Osteoporos Int. 2010, 21:399-408. 10.1007/s00198009-0962-6

14. Streubel PN, Ricci WM, Wong A, Gardner MJ: Mortality after distal femur fractures in elderly patients . Clin Orthop Relat Res. 2011, 469:1188-1196. 10.1007/s11999-010-1530-2

15. Myers P, Laboe P, Johnson KJ, Fredericks PD, Crichlow RJ, Maar DC, Weber TG: Patient mortality in geriatric distal femur fractures. J Orthop Trauma. 2018, 32:111-115. 10.1097/BOT.0000000000001078 